\title{
Galhas de insetos da Reserva Biológica Estadual da Praia do Sul (Ilha Grande, Angra dos Reis, RJ)
}

\author{
Valéria Cid Maia $^{1,2}$ \& Jussara Costa de Oliveira ${ }^{1}$ \\ ${ }^{1}$ Museu Nacional, Quinta da Boa Vista, São Cristóvão, CEP 20940-040, Rio de Janeiro, RJ, Brasil \\ ${ }^{2}$ Autor para correspondência: Valéria Cid Maia, e-mail: maiavcid@acd.ufrj.br
}

MAIA, V.C. \& OLIVEIRA, J.C. Insect galls of the Reserva Biológica Estadual da Praia do Sul (Ilha Grande, Angra dos Reis, RJ). Biota Neotrop. 10(4): http://www.biotaneotropica.org.br/v10n4/en/abstract?inventory+ bn04110042010.

\begin{abstract}
Thirty-six morphotypes of insect galls were recorded for the first time in the Reserva Biológica Estadual da Praia do Sul (Ilha Grande, Angra dos Reis, RJ), being the majority of them comprised of leaf galls (64\%). Stem and bud galls were also found (25 and 14\%, respectively). Myrtaceae were the plant family with the greatest richness of insect galls. Mikania sp. (Asteraceae) and Guapira opposita (Nyctaginaceae) were the super host plant species. The most important galling group was the Cecidomyiidae (Diptera), responsible for $75 \%$ of the gall morphotypes. Hemipteran and Lepidopteran galls were also recorded (11 and 2.8\%, respectively).

Keywords: Cecidomyiidae, Diptera, diversity, galls, restinga.
\end{abstract}

MAIA, V.C. \& OLIVEIRA, J.C. Galhas de insetos da Reserva Biológica Estadual da Praia do Sul (Ilha Grande, Angra dos Reis, RJ). Biota Neotrop. 10(4): http://www.biotaneotropica.org.br/v10n4/pt/abstract? inventory+bn04110042010.

Resumo: Trinta e seis morfotipos de galhas de insetos foram registrados pela primeira vez para a Reserva Biológica Estadual da Praia do Sul (Ilha Grande, Angra dos Reis, RJ), sendo a maioria foliar (64\%). Galhas desenvolvidas no caule e na gema também foram encontradas (25 e 14\%, respectivamente). As Myrtaceae representaram a família de planta com maior riqueza de galhas. Mikania sp. (Asteraceae) e Guapira opposita (Nyctaginaceae) foram as espécies vegetais super-hospedeiras. Os Cecidomyiidae (Diptera) destacaram-se como o principal grupo galhador, responsáveis por $75 \%$ dos morfotipos. Galhas de Hemiptera e Lepidoptera também foram registradas (11 e 2,8\%, respectivamente).

Palavras-chave: Cecidomyiidae, Diptera, diversidade, galhas, restinga. 


\section{Introdução}

As restingas fazem parte do Bioma Mata Atlântica, e podem ser definidas como longas faixas de depósitos arenosos marinhos, datados do Quaternário (Araújo 1992, Araújo et al. 1998, Scarano 2002). Inicialmente, as restingas ocupavam cerca de $80 \%$ da costa brasileira (Lacerda et al. 1993), mas devido à ação antrópica causada principalmente pela especulação imobiliária, vêm sofrendo grande perda de área.

As restingas compreendem uma considerável diversidade biológica (Rocha et al. 2004) e possuem uma comunidade vegetal característica (Lacerda et al. 1982, Rizzini 1992). As espécies de planta nelas encontradas apresentam um conjunto de adaptações relativas às condições extremas deste ambiente, como a elevada salinidade, a baixa disponibilidade de água e a intensa radiação solar (Scarano et al. 2001).

As restingas apresentam grande riqueza de galhas induzidas por insetos. No Brasil, na região sudeste, essas formações foram investigadas nos Estados do Rio de Janeiro (Maricá, Carapebus, Arraial do Cabo e Grumari), São Paulo (Bertioga) e Espírito Santo (Parque Paulo César Vinha) (Maia 2001, Monteiro et al. 1994, 2004, Maia \& Oliveira 2005, Maia et al. 2008, Bregonci et al. 2010). Para as demais localidades, não há informação disponível.

O objetivo deste trabalho é inventariar as galhas de insetos da Reserva Biológica Estadual da Praia do Sul (Ilha Grande, Angra dos Reis, RJ), contribuindo para o conhecimento da diversidade de galhadores nessa localidade e no Estado do Rio de Janeiro.

\section{Local de estudo}

A Reserva Biológica Estadual da Praia do Sul (RBEPS), com cerca de 3.600 ha, representa um importante remanescente de restinga preservada do Estado do Rio de Janeiro. Localiza-se na parte meridional da Ilha Grande, RJ (23 $10^{\prime}$ S e $\left.44^{\circ} 17^{\prime} \mathrm{W}\right)$, ocupando $18,2 \%$ de sua extensão. Abrange desde a ponta do Drago até Parnaóica, estendendo-se até a vertente das montanhas (Figuras 1 e 2). A temperatura do ar possui média anual de $22,5^{\circ} \mathrm{C}$, sendo fevereiro o mês mais quente, com temperaturas em torno de $25,7^{\circ} \mathrm{C}$, e julho o mês mais frio, com temperatura por volta de $19,6^{\circ} \mathrm{C}$. A flora dessa reserva inclui cinco comunidades vegetais (psamófila reptante da anteduna, mata de cordão arenoso, mata alagadiça de planície, manguezal e mata de encosta) e 302 espécies e variedades de plantas vasculares (Araújo \& Oliveira 1988).

O presente estudo foi realizado em duas comunidades: psamófila reptante da anteduna e na mata de cordão arenoso, cuja flora inclui 125 espécies de plantas vasculares, segundo Araújo \& Oliveira 1988.

\section{Material e Métodos}

Foram realizadas duas coletas na Reserva Biológica Estadual da Praia do Sul (RBEPS), em novembro de 2003 e em janeiro de 2004, na vegetação psamófila reptante e na mata de cordão arenoso. Cada coleta estendeu-se por dois dias consecutivos, perfazendo 8 horas diárias de investigação, ao longo da trilha de acesso à Praia do Sul.

As espécies vegetais foram vistoriadas à procura de galhas de insetos. Amostras das plantas galhadas, preferencialmente com flores e frutos foram herborizadas e identificadas por botânicos do Museu Nacional/UFRJ até o menor nível taxonômico possível. Todos os morfotipos de galhas encontrados foram fotografados em campo. Ramos vegetais com galhas foram retirados com auxílio de uma tesoura de poda e transportados em sacos plásticos etiquetados para o laboratório de Diptera do Museu Nacional.

No laboratório, uma parte da amostra de cada morfotipo de galha foi dissecada sob estereomicroscópio para observação do seu conteúdo e determinação do organismo galhador. Outra parte destinou-se à criação dos insetos galhadores. Amostras de cada morfotipo de galha foram individualmente acondicionadas em potes plásticos forrados com papel umedecido, fechados com tela fina e etiquetados. Esses potes eram vistoriados diariamente para verificação da emergência dos adultos. Pelo menos um exemplar de cada morfotipo de galha foi seco em estufa e depositado na coleção de galhas do Museu Nacional.

Os insetos obtidos a partir da criação e dissecação das galhas foram preservados em álcool etílico $70 \%$, acondicionados em microtubos de vidro fechados com batoque, etiquetados e incorporados à coleção de insetos galhadores do Museu Nacional.

Os galhadores foram identificados em nível de ordem, e no caso dos Cecidomyiidae (Diptera) no menor nível taxonômico possível, com base nas chaves de Gagné (1994) e nas descrições originais.

Todas as galhas foram caracterizadas morfologicamente quanto ao órgão de ocorrência na planta hospedeira, forma, coloração, número de câmaras internas, ausência ou presença de tricomas e inseto indutor.

\section{Resultados}

Foram encontrados 36 morfotipos de galhas de insetos em 22 espécies de plantas hospedeiras distribuídas em 16 famílias vegetais (Tabela 1), sendo 1,6 o número médio de morfotipos por espécie de planta hospedeira. As famílias de planta com maior riqueza de galhas foram Myrtaceae ( $n=9)$, Asteraceae (n=5) e Nyctaginaceae $(\mathrm{n}=4)$. Mikania sp. (Asteraceae) e Guapira opposita (Vell.) Reitz.

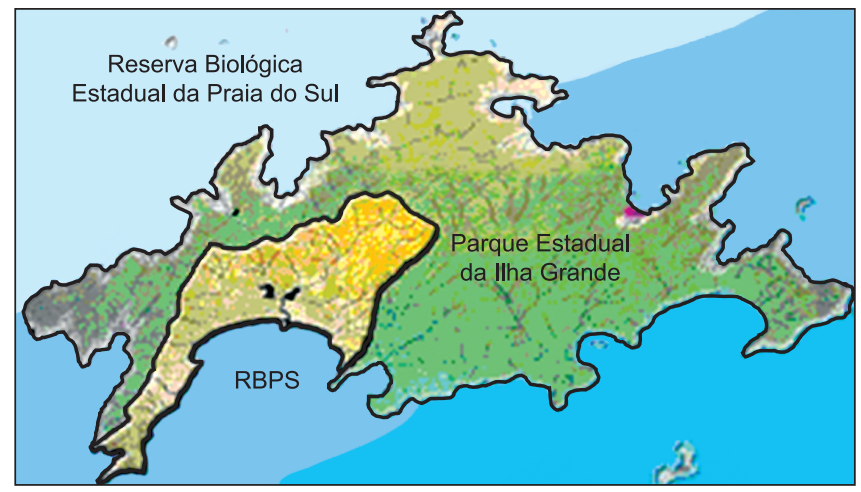

Figura 1. Mapa da Ilha Grande (Angra dos Reis, RJ).

Figure 1. Map of Ilha Grande (Angra dos Reis, RJ).

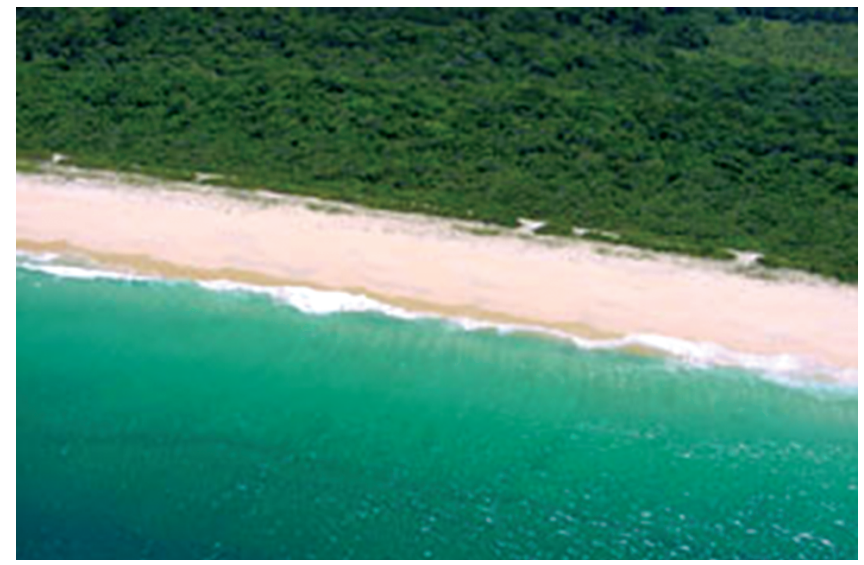

Figura 2. Praia do Sul (Ilha Grande, Angra dos Reis, RJ).

Figure 2. Praia do Sul (Ilha Grande, Angra dos Reis, RJ). 
Tabela 1. Distribuição do número de morfotipos de galhas de insetos por planta hospedeira na Reserva Biológica Estadual da Praia do Sul (Ilha Grande, Angra dos Reis, RJ).

Table 1. Distribution of the number of morphotypes of insect galls per host plant in the Reserva Biológica Estadual da Praia do Sul (Ilha Grande, Angra dos Reis, RJ).

\begin{tabular}{|c|c|c|}
\hline Família de planta & Espécie vegetal & $\mathrm{N}^{0}$ de morfotipos de galhas de insetos \\
\hline Anacardiaceae & Schinus terebinthifolius Raddi & 01 \\
\hline Aquifoliaceae & Ilex sp. & 01 \\
\hline Apocynaceae & Aspidosperma pyricollum Muell. Arg & 01 \\
\hline \multirow[t]{2}{*}{ Asteraceae } & Baccharis sp.1 & 01 \\
\hline & Mikania sp. & 04 \\
\hline Cactaceae & Selenicereus setaceus(SD) Berg & 01 \\
\hline Erythroxylaceae & Erythroxylum ovalifolium Peyr & 02 \\
\hline Euphorbiaceae & Dalechampia leandrii Baill. & 01 \\
\hline \multirow[t]{3}{*}{ Fabaceae } & Dalbergia ecastophylla L. Taub & 01 \\
\hline & Inga sp. & 01 \\
\hline & Não determinada & 01 \\
\hline Malpighiaceae & Byrsonima sericea DC. & 02 \\
\hline Melastomataceae & Não determinada & 01 \\
\hline Myrsinaceae & Rapanea sp. & 01 \\
\hline \multirow[t]{4}{*}{ Myrtaceae } & Myrciaria jaboticaba (Vell.) Berg & 02 \\
\hline & Eugenia uniflora L. & 02 \\
\hline & Eugenia sp. & 03 \\
\hline & Psidium cattleianum Sabine & 02 \\
\hline Nyctaginaceae & Guapira opposita (Vell.) Reitz. & 04 \\
\hline Ochnaceae & Ouratea cuspidata (St. Hill). Engl. & 02 \\
\hline Sapotaceae & Manilkara subsericea (Mart.) Dubard & 01 \\
\hline Smilacaceae & Smilax rufescens Griseb & 01 \\
\hline
\end{tabular}

(Nyctaginaceae) foram as espécies vegetais consideradas como "super-hospedeiras" (termo utilizado por Espírito-Santo \& Fernandes 2007 para designar espécies de plantas que suportam alta riqueza de galhas).

Cerca de $65 \%$ dos morfotipos das galhas ocorreram nas folhas. Galhas nos caules e gemas também foram encontradas, porém em proporções consideravelmente menores ( 25 e $14 \%$, respectivamente). Galhas em flores, frutos ou raízes adventícias não foram observadas, embora esses órgãos tenham sido vistoriados (Tabela 2). Todos os morfotipos ocorreram em um único órgão vegetal, com apenas duas exceções: as galhas cônicas em Mikania sp. (Asteraceae) e as galhas esferóides em Psidium cattleianum (Myrtaceae), com ocorrência nas folhas e caule e nas folhas e gema, respectivamente.

As galhas encontradas foram classificadas em simples e complexas, seguindo-se as definições de Möhn (1961). Os espessamentos fusiformes e esferóides do caule, da nervura e do pecíolo, as galhas circulares parenquimáticas e os enrolamentos da borda foliar foram considerados como galhas simples, enquanto que os demais morfotipos foram considerados complexos. As galhas simples foram as mais comuns, com $60 \%$ do total dos morfotipos. As galhas uniloculares tiveram amplo predomínio, assim como as glabras (ambas com cerca de $94 \%$ do total). A maioria dos morfotipos apresentou a mesma coloração do órgão vegetal hospedeiro (folha, pecíolo ou caule), com apenas duas exceções: as galhas foliares induzidas por Bruggmannia robusta Maia \& Couri, 1993 e por Contarinia sp. (Tabela 3). Ambas ocorreram em folhas de coloração verde, no entanto apresentaram coloração vermelha e marrom, respectivamente.

Os morfotipos de galhas foram induzidos por três ordens de insetos: Diptera (Cecidomyiidae); Hemiptera e Lepidoptera. Dentre esses, verificou-se um claro predomínio dos Cecidomyiidae, com $75 \%$ do total (Tabela 4). Galhas induzidas por Coleoptera, Thysanoptera e
Tabela 2. Distribuição do número de morfotipos de galhas de insetos por órgão da planta hospedeira na Reserva Biológica Estadual da Praia do Sul (Ilha Grande, Angra dos Reis, RJ).

Table 2. Distribution of the number of morphotypes of insect galls per plant organ in the Reserva Biológica Estadual da Praia do Sul (Ilha Grande, Angra dos Reis, RJ).

\begin{tabular}{lc}
\hline $\begin{array}{c}\text { Órgão da planta } \\
\text { hospedeira }\end{array}$ & $\begin{array}{c}\mathbf{N}^{\mathbf{0}} \text { de morfotipos de } \\
\text { galhas de insetos }\end{array}$ \\
\hline Folha & 23 \\
Caule & 09 \\
Gema & 05 \\
Flor & 0 \\
Fruto & 0 \\
Raízes adventícias & 0 \\
\hline
\end{tabular}

Hymenoptera não foram encontradas, embora essas ordens incluam representantes galhadores em outras restingas, como indicado por Monteiro et al. (1994) e Maia et al. (2008).

Todos os registros de galhas são inéditos para a Ilha Grande (RJ). Myrciaria jaboticaba (Vell.) Berg (Myrtaceae) é assinalada, pela primeira vez, como planta hospedeira de galhas de insetos.

\section{Discussão}

A diversidade de galhas de insetos na RBEPS (36 morfotipos) é menor que a das demais restingas da região sudeste (Tabela 5). Diversos fatores podem explicar essa diferença, tais como a composição florística (idade dos grupos taxonômicos e riqueza das espécies vegetais), o estado de preservação do ecossistema, a área 
Tabela 3. Distribuição dos morfotipos de galhas de insetos encontrados na Reserva Biológica Estadual da Praia do Sul (Ilha Grande, Angra dos Reis, RJ) por espécie de planta hospedeira e caracterização quanto à forma, cor, número de câmaras internas, presença de tricomas, órgão de ocorrência na planta e inseto galhador.

Table 3. Distribution of the morphotypes of insect galls found in the Reserva Biológica Estadual da Praia do Sul (Ilha Grande, Angra dos Reis, RJ) per host plant species and characterization by shape, color, number of internal chambers, presence of trichomes, plant organ and galling insect.

\begin{tabular}{|c|c|c|c|c|c|c|}
\hline $\begin{array}{c}\text { Planta } \\
\text { hospedeira }\end{array}$ & $\begin{array}{c}\text { Forma da } \\
\text { galha }\end{array}$ & Cor & $\begin{array}{c}\mathrm{N}^{0} \text { câmaras } \\
\text { internas }\end{array}$ & Tricomas & Órgão & Galhador \\
\hline Schinus terebinthifolius & circular (Figura 3) & verde & 01 & ausentes & folha & Hemiptera \\
\hline Ilex sp. & esférica (Figura 4) & verde & 01 & ausentes & gema & Cecidomyiidae \\
\hline Aspidosperma pyricollum & circular (Figura 5) & verde & 01 & ausentes & folha & Psyllidae (Hemiptera) \\
\hline Baccharis sp. & $\begin{array}{l}\text { fusiforme } \\
\text { (Figura 6) }\end{array}$ & verde & 01 & ausentes & folha & Não determinado \\
\hline \multirow[t]{4}{*}{ Mikania sp. } & $\begin{array}{l}\text { fusiforme } \\
\text { (Figura 7) }\end{array}$ & verde & 01 & ausentes & caule & $\begin{array}{l}\text { Mikaniadiplosis annulipes } \\
\text { Gagné, } 2001 \\
\text { Cecidomyiidae }\end{array}$ \\
\hline & $\begin{array}{l}\text { esferóide } \\
\text { (Figura 8) }\end{array}$ & verde & 01 & ausentes & folha & $\begin{array}{l}\text { Liodiplosis spherica } \\
\text { Gagné, } 2001 \\
\text { Cecidomyiidae }\end{array}$ \\
\hline & cilíndrica & verde & 01 & ausentes & folha & $\begin{array}{l}\text { Liodiplosis cylindrica } \\
\text { Gagné, } 2001 \\
\text { Cecidomyiidae }\end{array}$ \\
\hline & $\begin{array}{l}\text { cônica } \\
\text { (Figura 9) }\end{array}$ & verde & 01 & ausentes & folha/caule & $\begin{array}{l}\text { Liodiplosis conica } \\
\text { Gagné, } 2001 \\
\text { Cecidomyiidae }\end{array}$ \\
\hline Selenicereus setaceus & $\begin{array}{l}\text { esferóide } \\
\text { (Figura 10) }\end{array}$ & verde & várias & ausentes & caule & $\begin{array}{l}\text { Neolasioptera cerei } \\
\text { Rübsaamen, } 1905 \\
\text { Cecidomyiidae }\end{array}$ \\
\hline \multirow[t]{2}{*}{ Erythroxylum ovalifolium } & $\begin{array}{l}\text { cônica } \\
\text { (Figura 11) }\end{array}$ & verde & 01 & ausentes & gema & Cecidomyiidae \\
\hline & $\begin{array}{l}\text { enrolamento } \\
\text { marginal } \\
\text { (Figura 12) }\end{array}$ & verde & 01 & ausentes & folha & Cecidomyiidae \\
\hline Dalechampia leandrii & $\begin{array}{l}\text { emaranhado } \\
\text { (Figura 13) }\end{array}$ & verde & 01 & presentes & gema & $\begin{array}{l}\text { Schizomyiina } \\
\text { Cecidomyiidae }\end{array}$ \\
\hline Dalbergia ecastophylla & $\begin{array}{l}\text { discóide } \\
\text { (Figura 14) }\end{array}$ & verde & 01 & ausentes & folha & $\begin{array}{l}\text { Lopesia grandis Maia, } 2001 \\
\text { Cecidomyiidae }\end{array}$ \\
\hline Inga sp. & $\begin{array}{l}\text { esférica } \\
\text { (Figura 15) }\end{array}$ & verde & 01 & ausentes & folha & Cecidomyiidae \\
\hline Fabaceae não determinada & $\begin{array}{l}\text { linear } \\
\text { (Figura 16) }\end{array}$ & verde & 01 & ausentes & folha & Cecidomyiidae \\
\hline \multirow[t]{2}{*}{ Byrsonima sericea } & circular & verde & 01 & ausentes & folha & $\begin{array}{l}\text { Lasiopteridi } \\
\text { Cecidomyiidae }\end{array}$ \\
\hline & $\begin{array}{l}\text { esferóide } \\
\text { (Figura 17) }\end{array}$ & marrom & várias & ausentes & caule & Cecidomyiidae \\
\hline $\begin{array}{l}\text { Melastomataceae } \\
\text { não determinada }\end{array}$ & $\begin{array}{l}\text { fusiforme } \\
\text { (Figura 18) }\end{array}$ & marrom & 01 & ausentes & caule & Não determinado \\
\hline Rapanea sp. & $\begin{array}{l}\text { fusiforme } \\
\text { (Figura 19) }\end{array}$ & marrom & 01 & ausentes & caule & Lepidoptera \\
\hline \multirow[t]{2}{*}{ Myrciaria jaboticaba } & $\begin{array}{l}\text { enrolamento } \\
\text { marginal } \\
\text { (Figura 20) }\end{array}$ & verde & 01 & ausentes & folha & Cecidomyiidae \\
\hline & $\begin{array}{l}\text { cilíndrica } \\
\text { (Figura 21) }\end{array}$ & verde & 01 & ausentes & gema & Cecidomyiidae \\
\hline \multirow[t]{2}{*}{ Eugenia uniflora L. } & $\begin{array}{l}\text { fusiforme } \\
\text { (Figura 22) }\end{array}$ & marrom & 01 & ausentes & caule & Não determinado \\
\hline & $\begin{array}{l}\text { circular } \\
\text { (Figura 23) }\end{array}$ & verde & 01 & ausentes & folha & $\begin{array}{l}\text { Neolasioptera eugeniae } \\
\text { Maia, } 1993 \text { Cecidomyiidae }\end{array}$ \\
\hline
\end{tabular}


Galhas de insetos

Tabela 3. Continuação...

\begin{tabular}{|c|c|c|c|c|c|c|}
\hline $\begin{array}{c}\text { Planta } \\
\text { hospedeira }\end{array}$ & $\begin{array}{c}\text { Forma da } \\
\text { galha }\end{array}$ & Cor & $\begin{array}{c}\text { No câmaras }^{\text {internas }} \\
\end{array}$ & Tricomas & Órgão & Galhador \\
\hline \multirow[t]{3}{*}{ Eugenia sp. } & cilíndrica & verde & 01 & ausentes & folha & Cecidomyiidae \\
\hline & $\begin{array}{l}\text { fusiforme } \\
\text { (Figura 24) }\end{array}$ & marrom & 01 & ausentes & caule & Não determinado \\
\hline & $\begin{array}{l}\text { circular } \\
\text { (Figura 25) }\end{array}$ & verde & 01 & ausentes & folha & Cecidomyiidae \\
\hline \multirow[t]{2}{*}{ Psidium cattleianum } & $\begin{array}{l}\text { bipuncta } \\
\text { (Figura 26) }\end{array}$ & verde & 01 & ausentes & folha & Hemiptera \\
\hline & $\begin{array}{l}\text { esferóide } \\
\text { (Figura 27) }\end{array}$ & amarela & 01 & ausentes & folha/gema & Cecidomyiidae \\
\hline \multirow[t]{4}{*}{ Guapira opposita } & $\begin{array}{l}\text { circular } \\
\text { (Figura 28) }\end{array}$ & verde & 01 & ausentes & folha & $\begin{array}{l}\text { Bruggmannia elongata Maia } \\
\text { \& Couri, } 1993 \\
\text { Cecidomyiidae }\end{array}$ \\
\hline & $\begin{array}{l}\text { esférica } \\
\text { (Figura 29) }\end{array}$ & verm & 01 & presentes & folha & $\begin{array}{l}\text { Bruggmannia robusta Maia \& } \\
\text { Couri, } 1993 \text { Cecidomyiidae }\end{array}$ \\
\hline & $\begin{array}{l}\text { triangular } \\
\text { (Figura 30) }\end{array}$ & verde & 01 & ausentes & folha & $\begin{array}{l}\text { Bruggmannia acaudata } \\
\text { Maia, } 2004 \\
\text { Cecidomyiidae }\end{array}$ \\
\hline & $\begin{array}{l}\text { fusiforme } \\
\text { (Figura 31) }\end{array}$ & marrom & 01 & ausentes & caule & $\begin{array}{l}\text { Proasphondylia guapirae } \\
\text { Maia \& Couri, } 1993 \\
\text { Cecidomyiidae }\end{array}$ \\
\hline \multirow[t]{2}{*}{ Ouratea cuspidata } & $\begin{array}{l}\text { circular } \\
\text { (Figura 32) }\end{array}$ & amarela & 01 & ausentes & folha & Cecidomyiidae \\
\hline & $\begin{array}{l}\text { cilíndrica com } \\
\text { projeção apical } \\
\text { (Figura 33) }\end{array}$ & marrom & 01 & ausentes & folha & $\begin{array}{l}\text { Contarinia sp. } \\
\text { Cecidomyiidae }\end{array}$ \\
\hline Manilkara subsericea & $\begin{array}{l}\text { circular } \\
\text { (Figura 34) }\end{array}$ & verde & 01 & ausentes & folha & Cecidomyiidae \\
\hline Smilax rufescens & $\begin{array}{l}\text { cônica } \\
\text { (Figura 35) }\end{array}$ & verde & 01 & ausentes & folha & Hemiptera \\
\hline
\end{tabular}

Tabela 4. Distribuição do número de morfotipos de galhas por ordem do inseto galhador, na Reserva Biológica Estadual da Praia do Sul (Ilha Grande, Angra dos Reis, RJ).

Table 4. Distribution of the number of morphotypes of insect galls per galling insect order in the Reserva Biológica Estadual da Praia do Sul (Ilha Grande, Angra dos Reis, RJ).

\begin{tabular}{lc}
\hline Ordem do inseto galhador & $\mathbf{N}^{\mathbf{0}}$ de morfotipos de galhas \\
\hline Diptera (Cecidomyiidae) & 27 \\
Hemiptera & 4 \\
Lepidoptera & 1 \\
Coleoptera & 0 \\
Hymenoptera & 0 \\
Thysanoptera & 0 \\
Não determinado & 4 \\
\hline
\end{tabular}

ocupada, o grau de estresse hídrico, o distanciamento de uma restinga em relação às demais, entre outros. Esses fatores foram amplamente discutidos por Southwood (1960, 1961), Fernandes \& Price (1988), Price (1991), Price et al. (1998), Veldtman \& McGeoch (2003) e Fleck \& Fonseca (2007). Para determinar quais fatores poderiam explicar essa baixa diversidade, seria necessário realizar um estudo comparativo entre a flora e as condições abióticas das diferentes restingas, que não faz parte do escopo desse trabalho.
Tabela 5. Distribuição do número de morfotipos de galhas de insetos em diferentes áreas de restinga da região sudeste do Brasil. PEPCV - Parque Estadual Paulo César Vinha; RBEPS - Reserva Biológica Estadual da Praia do Sul.

Table 5. Distribution of the number of morphotypes of insect galls in restinga areas of the Southern region of Brazil. PEPCV - Parque Estadual Paulo César Vinha; RBEPS - Reserva Biológica Estadual da Praia do Sul.

\begin{tabular}{lc}
\hline Localidade & No de morfotipo de galhas de insetos \\
\hline Bertioga, SP & 233 \\
Grumari, RJ & 43 \\
Maricá, RJ & 72 \\
Arraial do Cabo, RJ & 41 \\
Carapebus, RJ & 62 \\
PEPCV, ES & 38 \\
RBEPS, RJ & 36 \\
\hline
\end{tabular}

Dados sobre a composição florística das restingas de Bertioga, Grumari, Maricá e RBEPS são conhecidos. Para essas localidades, são registradas 611, 193, 204 e 302 espécies vegetais (Martins et al. 2008, Oliveira \& Maia 2005, Silva \& Somer 1984, Araújo \& Oliveira 1988) e 233, 43, 72 e 36 morfotipos de galhas de insetos, respectivamente (Maia et al. 2008, Oliveira \& Maia 2005, Maia 2001) Esses dados corroboram a hipótese de que a riqueza das espécies vegetais influencia positivamente a riqueza de galhas. Embora para 

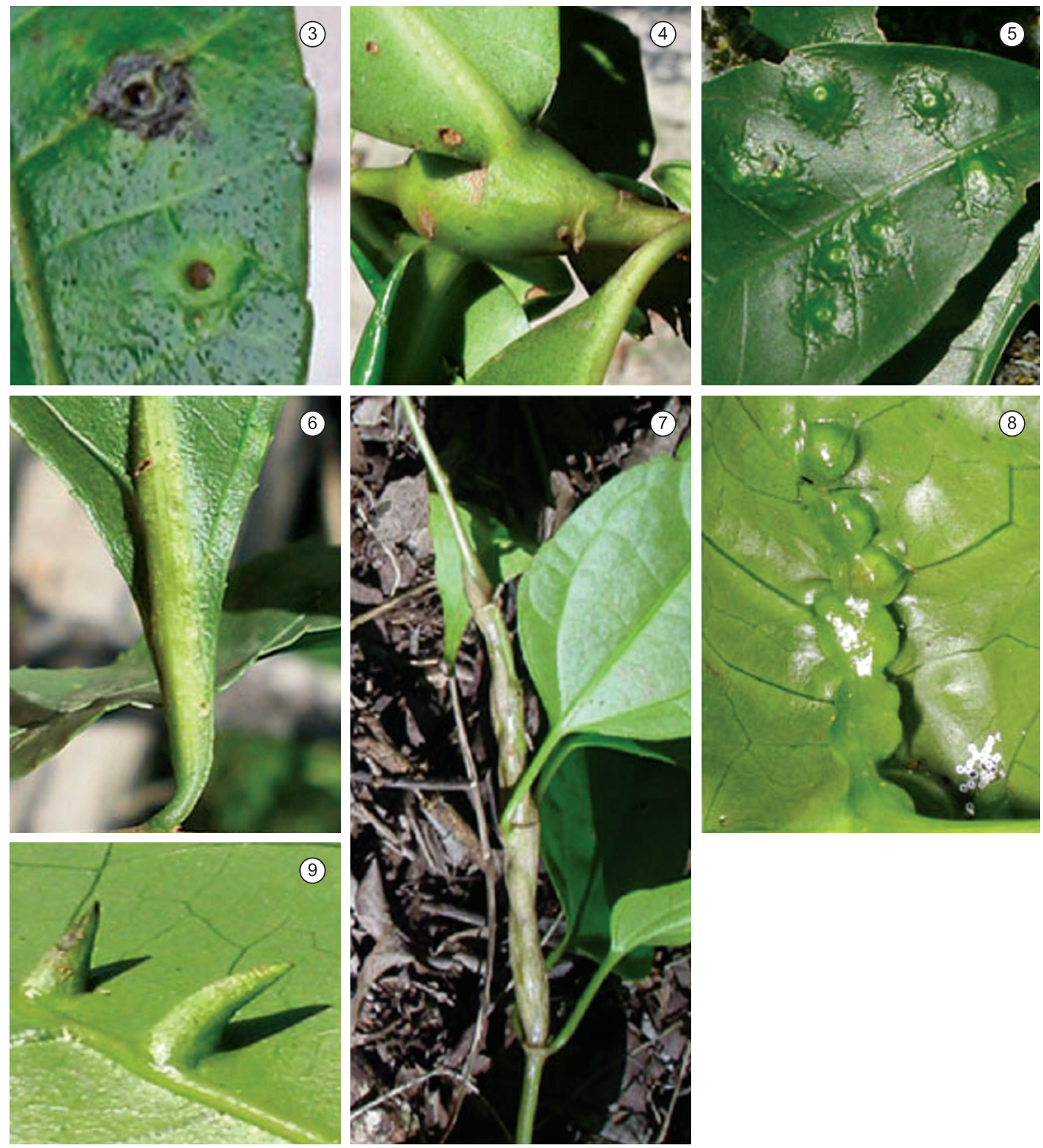

Figuras 3-9. Galhas de insetos da Reserva Biológica Estadual da Praia do Sul (Ilha Grande, Angra dos Reis, RJ). 3) Galha foliar em Schinus terebinthifolius; 4) Galha da gema em Ilex sp.; 5) Galha foliar em Aspidosperma pyricollum; 6) Galha foliar em Baccharis sp.; 7) Galha no caule em Mikania sp.; 8) Galha esferóide foliar em Mikania sp.; e 9) Galha cônica foliar em Mikania sp.

Figures 3-9. Insect galls from the Reserva Biológica Estadual da Praia do Sul (Ilha Grande, Angra dos Reis, RJ). 3) Leaf gall on Schinus terebinthifolius; 4) Bud gall on Ilex sp.; 5) Leaf gall on Aspidosperma pyricollum; 6) Leaf gall on Baccharis sp.; 7) Stem gall on Mikania sp.; 8) Spheroid leaf gall on Mikania sp.; and 9) Conical leaf gall on Mikania sp.

Tabela 6. Distribuição do número médio de morfotipos de galhas de insetos por espécie de planta em diferentes áreas de restinga da região Sudeste do Brasil. PEPCV - Parque Estadual Paulo César Vinha; RBEPS - Reserva Biológica Estadual da Praia do Sul).

Table 6. Distribution of the medium number of morphotypes of insect galls per plant species in restinga areas of the Southern region of Brazil. PEPCV - Parque Estadual Paulo César Vinha; RBEPS - Reserva Biológica Estadual da Praia do Sul.

\begin{tabular}{lc}
\hline Localidade & $\mathbf{N}^{\circ}$ médio de morfotipos de galhas de insetos por espécie de planta hospedeira \\
\hline Bertioga, SP & 1,9 \\
Grumari, RJ & 1,7 \\
Maricá, RJ & 1,7 \\
Arraial do Cabo, RJ & 1,5 \\
Carapebus, RJ & 2,0 \\
PEPCV, ES & 1,8 \\
RBEPS, RJ & 1,6 \\
\hline
\end{tabular}



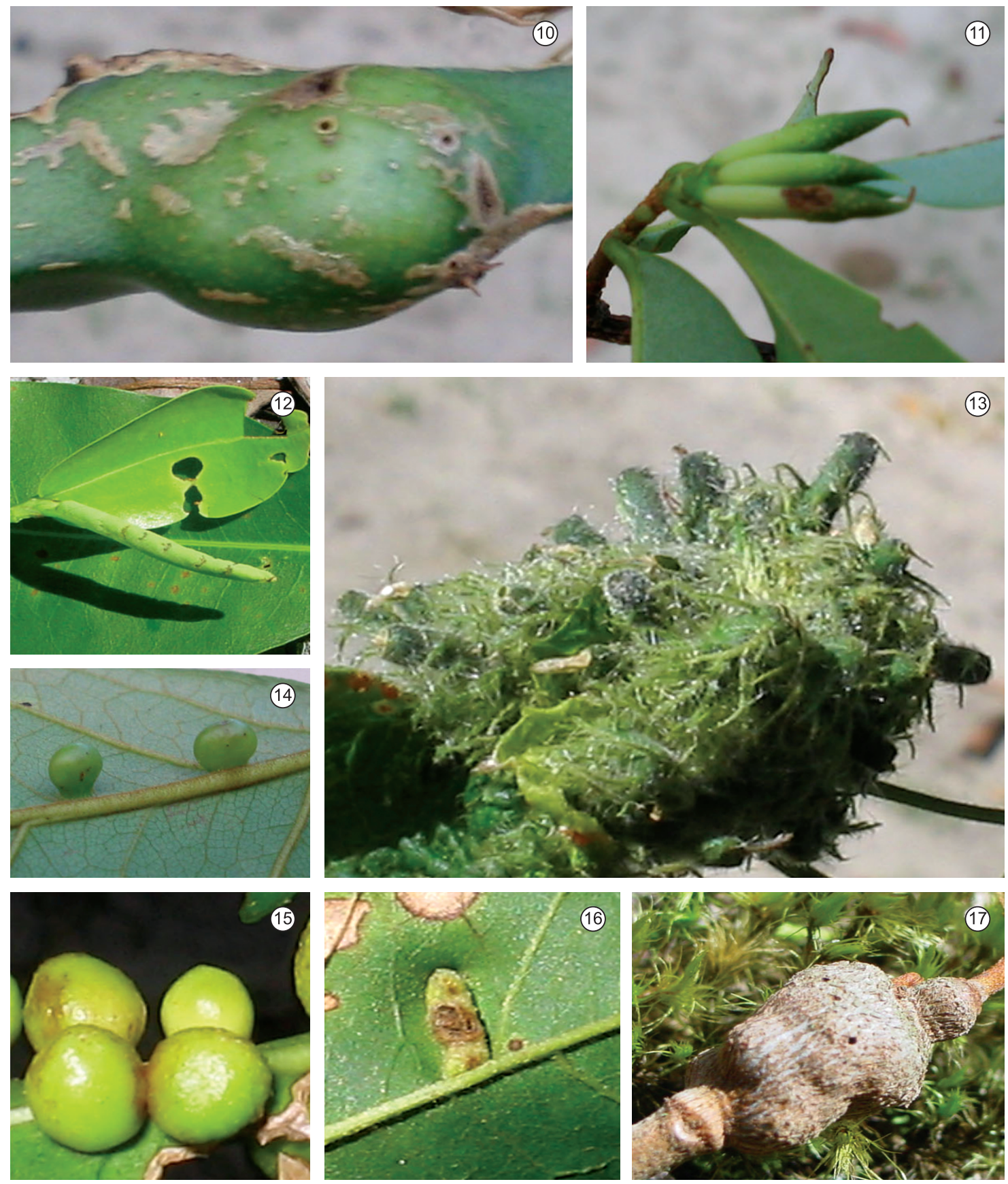

Figuras 10-17. Galhas de insetos da Reserva Biológica Estadual da Praia do Sul (Ilha Grande, Angra dos Reis, RJ). 10) Galha caulinar em Selenicereus setaceus; 11) galha da gema em Erythroxylum ovalifolium; 12) galha da borda foliar em Erythroxylum ovalifolium; 13) galha da gema em Dalechampia leandrii; 14) galha foliar em Dalbergia ecastophylla; 15) galha foliar em Inga sp.; 16) galha foliar em Fabaceae (não determinada); e 17) galha caulinar em Byrsonima sericea.

Figures 10-17. Insect galls from the Reserva Biológica Estadual da Praia do Sul (Ilha Grande, Angra dos Reis, RJ). 10) Stem gall on Selenicereus setaceus; 11) bud gall on Erythroxylum ovalifolium; 12) marginal leaf gall on Erythroxylum ovalifolium; 13) bud gall on Dalechampia leandrii; 14) leaf gall on Dalbergia ecastophylla; 15) leaf gall on Inga sp.; 16) leaf gall on Fabaceae (não determinada); and 17) stem gall on Byrsonima sericea.

a RBEPS haja 302 espécies de plantas registradas, as coletas de galhas foram realizadas apenas na comunidade psamófila reptante da anteduna e na mata do cordão arenoso, que incluem em conjunto 125 espécies de plantas.

O número médio de morfotipos de galhas por espécie de planta hospedeira foi 1,6. Valores similares foram encontrados para todas as outras áreas de restinga já estudadas na região sudeste (Tabela 6). Este fato é bastante interessante, sugerindo haver uma limitação no número de espécies galhadoras sustentadas por espécie de planta hospedeira.

As Myrtaceae representaram a família de planta com maior riqueza de galhas na RBEPS. O mesmo resultado foi encontrado para as restingas de Bertioga, Maricá, Arraial do Cabo e Carapebus por Maia et al. (2008), Monteiro et al. (1994) e Maia (2001). Em Grumari e no Parque Estadual Paulo César Vinha, as Myrtaceae ocuparam o segundo lugar (Oliveira \& Maia 2005, Bregonci et al. 2010). Portanto, 

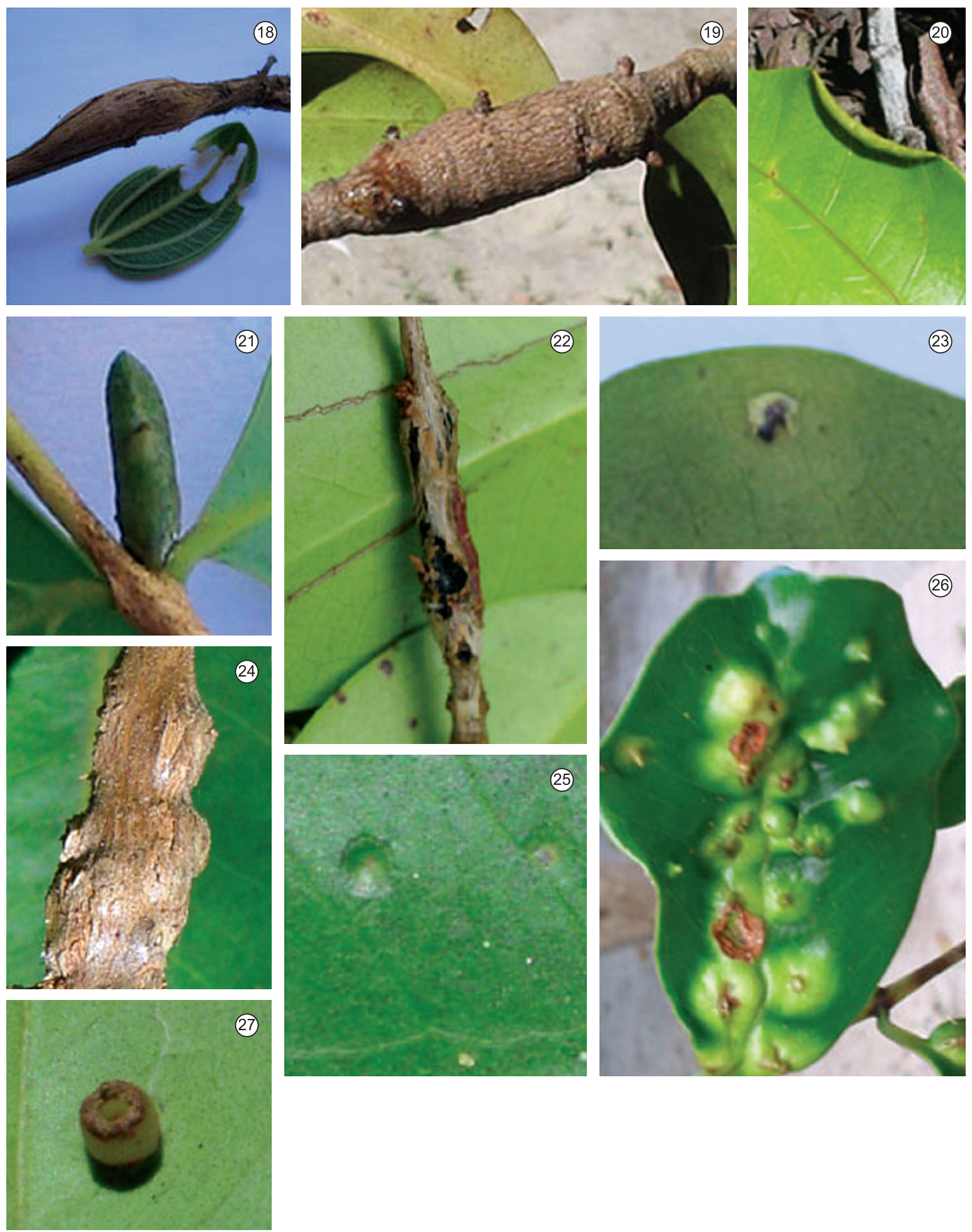

Figuras 18-27. Galhas de insetos da Reserva Biológica Estadual da Praia do Sul (Ilha Grande, Angra dos Reis, RJ). 18) Galha caulinar em Melastomataceae (não determinada); 19) galha caulinar em Rapanea sp.; 20) galha da borda foliar em Myrciaria jaboticaba; 21) galha da gema em Myrciaria jaboticaba; 22) galha caulinar em Eugenia uniflora; 23) galha foliar em Eugenia uniflora; 24) galha caulinar em Eugenia sp.; 25) galha circular foliar em Eugenia sp.; 26) galha triangular foliar em Psidium cattleianum; e 27) galha esferóide foliar em Psidium cattleianum.

Figures 18-27. Insect gall from the Reserva Biológica Estadual da Praia do Sul (Ilha Grande, Angra dos Reis, RJ). 18) Stem gall on Melastomataceae (não determinada); 19) Stem gall on Rapanea sp.; 20) Marginal leaf gall on Myrciaria jaboticaba; 21) Bud gall on Myrciaria jaboticaba; 22) Stem gall on Eugenia uniflora; 23) Leaf gall on Eugenia uniflora; 24) Stem gall on Eugenia sp.; 25) Circular leaf gall on Eugenia sp.; 26) Triangular leaf gall on Psidium cattleianum; and 27) Spheroid leaf gall on Psidium cattleianum. 

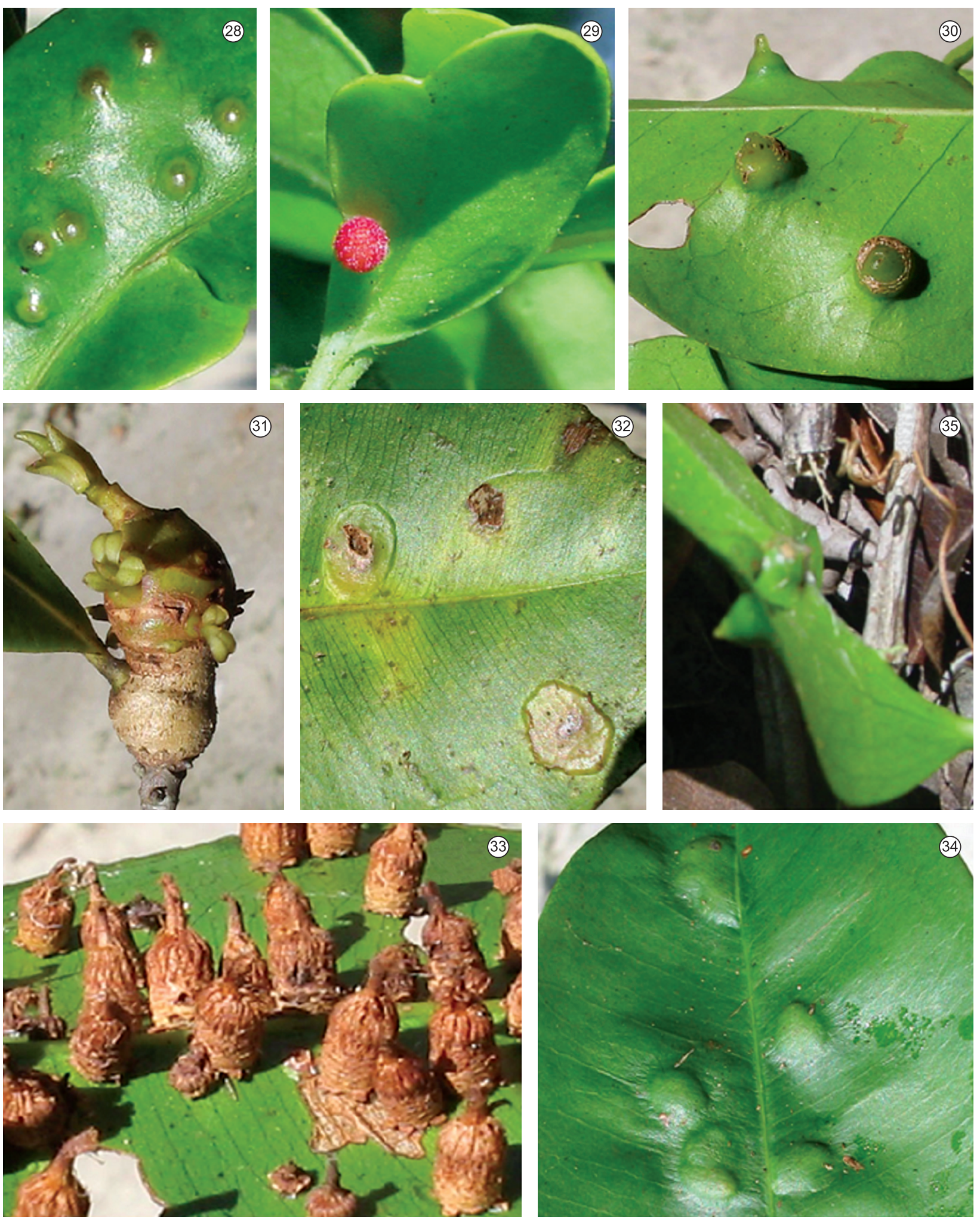

Figuras 28-35. Galhas de insetos da Reserva Biológica Estadual da Praia do Sul (Ilha Grande, Angra dos Reis, RJ. 28) Galha circular em Guapira opposita; 29) galha esférica em Guapira opposita; 30) galha triangular em Guapira opposita; 31) galha caulinar em Guapira opposita; 32) galha foliar circular em Ouratea cuspidata; 33) galha foliar cilíndrica com projeção apical em Ouratea cuspidata; 34) galha foliar em Manilkara subsericea; e 35) galha foliar em Smilax rufescens.

Figures 28-35. Insect galls from the Reserva Biológica Estadual da Praia do Sul (Ilha Grande, Angra dos Reis, RJ. 28) Circular gall on Guapira opposita; 29) spherical gall on Guapira opposita; 30) triangular gall on Guapira opposita; 31) stem gall on Guapira opposita; 32) circular leaf gall on Ouratea cuspidata; 33) cylindrical leaf gall, with an apical projection on Ouratea cuspidata; 34) leaf gall on Manilkara subsericea; and 35) leaf gall on Smilax rufescens. 
Tabela 7. Distribuição do número total de morfotipos de galhas de insetos em áreas de restinga da região sudeste e do número de morfotipos assinalados para a Reserva Biológica Estadual da Praia do Sul (RBEPS), Angra dos Reis, RJ por espécie de planta hospedeira.

Table 7. Distribution of the total number of morphotypes of insect galls in restinga areas of the Southern region and number of morphotypes recorded from the Reserva Biológica Estadual da Praia do Sul (RBEPS), Angra dos Reis, RJ.

\begin{tabular}{lcc}
\hline Planta hospedeira & $\mathbf{N}^{\mathbf{0}}$ total de morfotipos de galhas de insetos & $\mathbf{N}^{0}$ de morfotipos assinalados para RBEPS \\
\hline Schinus terebinthifolius & 02 & 01 \\
Aspidosperma pyricollum & 02 & 01 \\
Selenicereus setaceus & 01 & 01 \\
Erythroxylum ovalifolium & 05 & 02 \\
Dalechampia leandrii & 01 & 01 \\
Dalbergia ecastophylla & 01 & 01 \\
Byrsonima sericea & 04 & 02 \\
Eugenia uniflora & 06 & 02 \\
Myrciaria jaboticaba & 0 & 02 \\
Psidium cattleianum & 05 & 01 \\
Guapira opposita & 06 & 04 \\
Ouratea cuspidata & 01 & 02 \\
Manilkara subsericea & 03 & 01 \\
Smilax rufescens & 03 & 01
\end{tabular}

diferentes estudos indicam as Myrtaceae como uma das principais famílias de planta hospedeira de galhas de insetos. Esse resultado era esperado, uma vez que as Myrtaceae estão muito bem representadas nas restingas e contribuem significativamente para a caracterização de sua flora (Rizzini 1979).

Guapira opposita (Vell.) Reitz. destacou-se na RBEPS como a espécie de planta "super-hospedeira". Nas demais restingas da região sudeste do Brasil, G. opposita também tem posição de destaque (Maia et al. 2008, Monteiro et al. 1994, Maia 2001), exceto no Parque Estadual Paulo César Vinha (PEPCV) e em Grumari, onde Manilkara subsericea (Mart.) Dubard (Sapotaceae) e Erythroxylum ovalifolium Peyr (Erythroxylaceae) são as espécies vegetais super-hospedeiras, respectivamente. Em PECPV, G. opposita apresenta apenas um morfotipo de galha; já em Grumari, não há registro dessa espécie de planta.

As galhas foliares predominaram. Esse mesmo padrão é observado não só nas demais restingas da região sudeste, como também em todos os outros biomas brasileiros e em todas as regiões biogegráficas. Tratase, portanto, de um padrão mundial conforme apontado por (Mani 1964). Não foram encontradas galhas nos órgãos reprodutivos das plantas hospedeiras que estavam férteis durante o trabalho de campo. Nas demais restingas da região sudeste, a ocorrência de galhas em flores e/ou frutos foi assinalada, exceto no PEPCV. Galhas em flores e frutos são menos comuns, e sua visualização é mais difícil, porque muitas vezes as modificações restringem-se à estrutura interna do órgão atacado, não ocorrendo nenhuma modificação externa. Por isso, essas galhas podem facilmente passar despercebidas, se a metodologia de coleta não incluir dissecções das flores e frutos encontrados. Além disso, a disponibilidade de flores e frutos é menos frequente ao longo dos meses do ano, portanto esses órgãos representam um recurso menos constante que as folhas.

Os galhadores encontrados pertencem a apenas três ordens: Diptera (Cecidomyiidae), Hemiptera e Lepidoptera. Esse resultado mostra uma baixa riqueza de ordens de insetos galhadores, uma vez que galhas induzidas por Coleoptera, Hymenoptera e Thysanoptera também são conhecidas de outras restingas (Monteiro et al. 1994, Maia et al. 2008). Das seis ordens de insetos que incluem representantes galhadores (Diptera, Lepidoptera, Coleoptera, Hymenoptera, Hemiptera e Thysanoptera), todas estão presentes em Maricá (Monteiro et al. 1994, Maia 2001), cinco estão presentes em Bertioga (Maia et al. 2008) e em Grumari (Oliveira \& Maia 2005), e apenas duas no PEPCV (Bregonci et al. 2010). No entanto, em todas as localidades, as galhas de Cecidomyiidae predominaram, com percentuais variando de 75 a 91\% (Monteiro et al. 1994, Maia 2001, Oliveira \& Maia 2005, Maia et al. 2008, Bregonci et al. 2010).

Quase todas as espécies vegetais assinaladas já eram conhecidas por apresentar galhas de insetos. No entanto, o número de morfotipos de galhas encontrados em algumas espécies hospedeiras diferiu do observado em outras restingas (Tabela 7). As seguintes plantas apresentaram menor riqueza de morfotipos: Schinus terebinthifolius Raddi (Anacardiaceae), Aspidosperma pyricollum Muell. Arg (Apocynaceae), Erythroxylum ovalifolium Peyr (Erythroxylaceae), Byrsonima sericea DC. (Malpighiaceae), Eugenia uniflora L. (Myrtaceae), Psidium cattleianum Sabine (Myrtaceae), Guapira opposita (Vell.) Reitz. (Nyctaginaceae), Manilkara subsericea (Mart.) Dubard (Sapotaceae) e Smilax rufescens Griseb (Smilacaceae). Uma única espécie de planta apresentou maior riqueza de morfotipos na RBEPS: Ouratea cuspidata (St. Hill). Engl. (Sapotaceae). A ocorrência de galhas de insetos em Myrciaria jaboticaba (Vell.) Berg (Myrtaceae) é registrada pela primeira vez. Trata-se de uma espécie de planta introduzida por ação humana nas proximidades das instalações da FEEMA.

\section{Conclusões}

A riqueza de galhas de insetos na RBEPS foi mais baixa em comparação a outras áreas de restinga da região sudeste do Brasil. No entanto, o número médio de morfotipos por espécie de planta hospedeira foi similar às demais.

As Myrtaceae representaram a família de planta com maior riqueza de galhas de insetos e Guapira opposita (Vell.) Reitz. (Nyctaginaceae) a espécie vegetal super-hospedeira, como já verificado em outras restingas.

A maioria dos morfotipos é foliar, unilocular e glabra, com ocorrência em apenas um único órgão vegetal, como observado nas demais restingas estudadas.

A guilda dos insetos galhadores é menos diversificada na RBEPS do que na maioria das outras áreas de restinga, incluindo representantes de apenas três ordens: Diptera, Hemiptera e Lepidoptera. As galhas de Cecidomyiidae (Diptera) predominaram na RBEPS, como em qualquer outra localidade do mundo.

Os 36 registros de galhas são novos para a Ilha Grande (RJ) e um novo registro de espécie de planta hospedeira, Myrciaria jaboticaba foi assinalado. 


\section{Agradecimentos}

Ao Sr. Antônio Clóvis Britto de Araújo (Museu Nacional/UFRJ) pelas fotografias, à Dra. Norma Crud Maciel (FEEMA) pelos contatos que viabilizaram o trabalho de campo, aos botânicos do Museu Nacional/UFRJ pela identificação das plantas e à FEEMA pela infraestrutura oferecida.

\section{Referências Bibliográficas}

ARAÚJO, D.S.D \& OLIVEIRA, R.R. 1988. Reserva Biológica Estadual da Praia do Sul (Ilha Grande, Estado do Rio de Janeiro): lista preliminar da flora. Acta Bot. Bras. 1(2):83-94. Supl.

ARAÚJO, D.S.D. 1992. Vegetation types of sandy coastal plains of tropical Brazil: a first aproximation. In Coastal plant communities of Latin America (U. Seelingere, ed.). Academic Press, San Diego, p.337-347.

ARAÚJO, D.S.D., SCARANO, F.R., SÁ, C.F.C., KURTZ, B.C., ZALUAR, H.L.T., MONTEZUMA, R.C.M. \& OLIVEIRA, R.C. 1998. Comunidades vegetais do Parque Nacional da Restinga de Jurubatiba. In Ecologia das Lagoas Costeiras do Parque Nacional da Restinga de Jurubatiba e do município de Macaé (RJ) (F.A. Esteves, ed.). Universidade Federal do Rio de Janeiro, Rio de Janeiro, p.39-62.

BREGONCI, J.M., POLYCARPO, P.V. \& MAIA, V.C. 2010. Insect galls of the Parque Estadual Paulo César Vinha (Guarapari, ES, Brazil). Biota Neotrop. 10 (1):265-274.

ESPÍRITO-SANTO, M.M. \& FERNANDES, G.W. 2007. How many species of Gall-inducing insects are there on Earth and where are they? Ann. Entomol. Soc. Am. 100(2):95-99.

FERNANDES, G.W. \& PRICE, P.W. 1988. The adaptative significance of insect gall distribution survivorship of species in xeric and mesic habitats. Oecologia 90:14-20.

FLECK, T. \& FONSECA, C.R. 2007. Hipóteses sobre a riqueza de insetos galhadores: uma revisão considerando os níveis intra-específico, interespecífico e de comunidade. Neot. Biol. Conserv. 2(1):36-45.

GAGNÉ, R.J. 1994. The Gall Midge of the neotropical Region. Cornell University Press, Ithaca, N.Y., 352p.

LACERDA, L.D., ARAÚJO, D.S.D. \& MACIEL, N.C. 1982. Restingas Brasileiras: uma bibliografia. Universidade Federal do Rio de Janeiro, Rio de Janeiro.

LACERDA, L.D., ARAÚJO, D.S.D. \& MACIEL, N.C. 1993. Dry coastal ecosystems of the tropical Brazilian coast. In: Dry coastal ecosystems: Africa, America, Asia, Oceania. Amsterdam. (E. van der Maarel, ed.). Elsevier, p.477-493.

MAIA, V.C. \& OLIVEIRA, J.C. 2005. Ocorrência e caracterização de galhas de insetos na restinga de Grumari (Rio de Janeiro, RJ, Brasil). Arq. Mus. Nac. 63(4):669-675.

MAIA, V.C. 2001. The gall midges (Diptera, Cecidomyiidae) from three restingas of Rio de Janeiro State, Brazil. Revista Brasileira de Zoologia 18(2):583-629.

MAIA, V.C., MAGENTA, M.A.G. \& MARTINS, S.E. 2008. Ocorrência e caracterização de galhas de insetos em áreas de restinga de Bertioga (São Paulo, Brasil). Biota Neot. 8(1): http:/www.biotaneotropica.org.br/ v8n1/pt (22/12/2010).
MANI, M.S. 1964. Ecology of plant galls. Dr. W. Junk Publisher, Holanda, 434p.

MARTINS, S.E., ROSSI, L., SAMPAIO, P.S.P. \& MAGENTA, M.A.G. 2008. Caracterização florística de comunidades vegetais de restinga em Bertioga, SP, Brasil. Acta Bot. Bras. 22(1):249-274.

MÖHN, E. 1961. Gallmücken (Diptera, Itonididae) aus El Salvador. 4.Zur Phylogenie der Asphondyliidi der neotropischen und holarktischen region. Senck. Biol. 42(3):131-330.

MONTEIRO, R.F., FERRAZ, F.F.F., MAIA, V.C. \& AZEVEDO, M.A.P. 1994. Galhas entomógenas em restingas. Uma abordagem preliminar. In III Simpósio de Ecossistemas da Costa Brasileira: subsídios a um gerenciamento ambiental (S. Watanabe, Coord.). ACIESP, São Paulo, v. 3, p. 210-220.

MONTEIRO, R.F., ODA, R.A.M., NARAHARA, K.L. \& CONSTANTINO, P.A.L. 2004. Galhas: diversidade, especificidade e distribuição. In Pesquisas de longa duração na Restinga de Jurubatiba: ecologia, história natural e conservação (C.F.D. Rocha, F.A. Esteves \& F.R. Scarano, Org.). Rima, São Carlos, 376p.

OLIVEIRA, J.C. \& MAIA, V.C. 2005. Ocorrência e Caracterização de galhas de insetos na Restinga de Grumari (Rio de Janeiro, RJ, Brasil). Arq. Mus. Nac. 63(4):669-675.

PRICE, P.W. 1991. The plant vigor hypothesis and herbivore attack. Oikos 62(2):244-251.

PRICE, P.W., FERNANDES, G.W., LARA, A.C.F., BRAWN, J., BARRIOS, H., WRIGHT, M.G., RIBEIRO, S.P. \& ROTHCLIFF, N. 1998. Global patterns in local number of insect galling species. J. Biogeogr. 25:581-591.

RIZZINI, C.T. 1979. Tratado de fitogeografia do Brasil. v.2. Aspectos ecológicos. Hucitec/Edusp, São Paulo.

RIZZINI, C.T. 1992. Tratado de fitogeografia do Brasil: aspectos ecológicos e florísticos. Âmbito Cultural Edições, São Paulo.

ROCHA, C.F.D., NUNES-FREITAS, A.F., ROCHA-PESSÔA, T.C. \& COGLIATTI-CARVALHO, L. 2004. Habitat disturbance in Brazilian Coastal sand dune vegetation and present richness and diversity of bromeliad species. Vidalia 2(2):50-56.

SCARANO, F.R. 2002. Strucuture, function and floristic relationships of plant communities in stressful habitats marginal to the Brazilian Atlantic Rainforest. Ann. Bot. 90(4):517-524.

SCARANO, F.R., DUARTE, H.M., RIBEIRO, K.T., RODRIGUES, P.J.F.P \& BARCELLOS, E.M.B. 2001. Four sites contrasting environmental stress in southeastern Brazil: relations of species, life form diversity, and geographic distribution to ecophysiological parameters. Bot. J. Linn. Soc. 136(4):345-364.

SILVA, J.G. \& SOMNER, G.V. 1984. A vegetação da restinga na Barra de Maricá, RJ. In Restingas. Origem, Processo e Estrutura (L.D. Lacerda, D.S.D. Araujo, R. Cerqueira \& B. Turcq, ed.) CEUFF, Niterói, RJ, p.217-225.

SOUTHWOOD, T.R. 1960. The abundance of Hawaiian trees and number of their associated insects and their host plants. Proc. Haw. Ent. Soc. 17:299-303.

SOUTHWOOD, T.R. 1961. The number of species of insects associated with various trees. J. Anim. Ecol. 30:1-8.

VELDTMAN, R. \& MCGEOCH, M.A. 2003. Galling-insect species richness along a non-scleromorphic vegetation gradient in South Africa: a test of the hygrothermal stress hypothesis. Aust. J. Ecol. 28, 1-13. 
